Communications in Physics, Vol. 27, No. 3 (2017), pp. 205-214

DOI:10.15625/0868-3166/27/3/10653

\title{
EDGE EFFECTS OF TRUNCATED DIRAC SOLITONS IN BINARY WAVEGUIDE ARRAYS
}

\author{
TRAN XUAN TRUONG ${ }^{\dagger}$, NGUYEN MINH HUE AND PHUNG DINH HOAT \\ Department of Physics, Le Quy Don University, \\ 236 Hoang Quoc Viet, Hanoi, Vietnam \\ ${ }^{\dagger}$ E-mail: tranxtr@gmail.com
}

Received 05 September 2017

Accepted for publication 26 September 2017

Published 31 September 2017

\begin{abstract}
We investigate the edge effects of the optical analogue of the quantum relativistic Dirac solitons in binary waveguide arrays with Kerr nonlinearity when one tail of the Dirac soliton is truncated. We show that if the outermost waveguide of the binary waveguide array hosts the intense component of the truncated Dirac soliton, then Dirac soliton will be repeatedly bent towards the binary waveguide array edge. In the contrast, if the outermost waveguide of the binary waveguide array hosts the weak component of the truncated Dirac soliton, then Dirac soliton will be pushed away from the binary waveguide array edge. To the best of our knowledge, these unique features have not been found in any other systems.
\end{abstract}

Keywords: nonlinear optics, binary waveguide array, Dirac soliton.

Classification numbers: 42.65.Tg, 42.81.Dp, 42.82.Et.

\section{INTRODUCTION}

Waveguide arrays (WAs) have attracted great attention because they can be used to simulate the evolution of a non-relativistic quantum mechanical particle in a periodic potential $[1,2]$. Many fundamental phenomena in non-relativistic classical and quantum mechanics such as Bloch oscillations [3,4] and Zener tunneling [5,6] have been investigated by using WAs. Recently, it was shown that several important nonlinear phenomena usually associated to fiber optics (such as the emission of resonant radiation from solitons and soliton self-wavenumber shift) can also take place in WAs, but in the spatial domain rather than in the temporal domain [7,8]; and the supercontinuum in both frequency and wave number domains can be generated in nonlinear WAs [9].

(C)2017 Vietnam Academy of Science and Technology 
Binary waveguide arrays (BWAs) have also been intensively used to mimic relativistic phenomena typical of quantum field theory, such as Klein tunneling [10,11], Zitterbewegung (trembling motion of a free Dirac electron) [12,13], and fermion pair production [14], which all emerge from the properties of the Dirac equation [15]. Quite recently, it has been shown in Ref. [16]that at the interface of two BWAs one can create the optical analogue of a special state, known in the quantum field theory as a Jackiw-Rebbi (JR) solution [17]. The JR state is well known for predicting the charge fractionalisation phenomenon which is fundamental to the fractional quantum Hall effect [18]. The JR state is also well known for the topological nature of its zero-energy solution and can be interpreted as a precursor to topological insulators which have attracted much interest recently $[19,20]$. Topological photonics can potentially play a crucial role in the development of robust and practical optical circuits for unidirectional transport of light [21,22].

The discrete gap solitons in BWAs in the classical context have been investigated earlier both numerically [23-28] and experimentally [29]. Recently, the explicit suggestion to use BWAs to simulate a quantum nonlinear Dirac equation has been put forward for the first time in Ref. [30] where the gap solitons in BWAs have been shown to be connected to Dirac solitons (DSs) in a nonlinear extension of the relativistic one-dimensional (1D) Dirac equation describing the dynamics of a freely moving relativistic particle. Other soliton solutions have been found for the nonlinear 1D Dirac equation [31], but with a different kind of nonlinearity, in the context of quantum field theory. The 1D DS stability, its dynamics and different scenarios of soliton interaction have been systematically investigated in Ref. [32]. The formation and dynamics of two-dimensional DSs in square binary waveguide lattices have been investigated in Ref. [33]. The higher-order Dirac solitons in BWAs have been studied in Ref. [34]. In Ref. [16] it has been shown that one can effectively generate Jackiw-Rebbi states starting from Dirac solitons. Although there is currently no evidence for fundamental quantum nonlinearities, nonlinear versions of the Dirac equation have been studied since a long time. One of the earlier extensions was investigated by Heisenberg [35] in the context of field theory and was motivated by the question of mass. In the quantum mechanical context, nonlinear Dirac equations have been used as effective theories in atomic, nuclear and gravitational physics [36-39]. To this regard, BWAs can offer a unique platform to simulate nonlinear extensions of the Dirac equation when light with high light intensities are used. One of these possibilities is to use BWAs as a classical simulator of the Dirac equation to mimic the two-body Dirac model, i.e. the Dirac equation for two interacting relativistic particles, which has attracted interest of researchers since the early days of quantum mechanics [40,41].

In this paper we investigate the unique edge effects of Dirac solitons in BWAs where one tail of the DS is truncated. We show that if the outermost waveguide of the BWA hosts the intense component of the truncated DS, then DS will be repeatedly bent towards the BWA edge. In the contrast, if the outermost waveguide of the BWA hosts the weak component of the truncated DS, then DS will be pushed away from the BWA edge. To the best of our knowledge, these unique features have not been found in any other systems.

The paper is organized as follows. In Sections II, as a starting point, we give the theoretical background of DSs in BWAs. Then, in Section III, we investigate the edge effects of DSs in BWAs. In Section IV, we study in detail the first touching distance of the DSs in the case where they are bent towards the BWA edge. Finally, in Section V we summarize our results and finish with concluding remarks. 


\section{THEORETICAL BACKGROUND OF DIRAC SOLITONS IN BWAS}

Light propagation in a binary array of Kerr nonlinear waveguides can be described, in the continuous-wave regime, by the following dimensionless coupled-mode equation (CME) [23]:

$$
i \frac{d a_{n}(z)}{d z}=-c\left[a_{n+1}(z)+a_{n-1}(z)\right]+(-1)^{n} \sigma a_{n}-\gamma\left|a_{n}(z)\right|^{2} a_{n}(z),
$$

where $a_{n}$ is the electric field amplitude in the $n$th waveguide, $\mathrm{z}$ is the longitudinal spatial coordinate, $2 \sigma$ and care the propagation mismatch and the coupling coe?ient between two adjacent waveguides of the array, respectively, and $\gamma$ is the nonlinear coe?ient of the waveguides. In the dimensionless form, in general, one can normalize variables in the above equation such that both $\gamma$ and $c$ are equal to unity. However, throughout this work we will keep these parameters explicitly in Eq. (1). The form of the analytical fundamental DS solution to the CME investigated in [30] is following:

$$
\left[\begin{array}{l}
a_{2 n}(z) \\
a_{2 n-1}(z)
\end{array}\right]=\left[\begin{array}{l}
i^{2 n} \frac{2 c}{n_{0} \sqrt{\sigma \gamma}} \operatorname{sech}\left(\frac{2 n}{n_{0}}\right) e^{i z\left(\frac{2 c^{2}}{n_{0}^{2} \sigma}-\sigma\right)} \\
i^{2 n} \frac{2 c^{2}}{n_{0}^{2} \sigma \sqrt{\sigma \gamma}} \operatorname{sech}\left(\frac{2 n-1}{n_{0}}\right) \tanh \left(\frac{2 n-1}{n_{0}}\right) e^{i z\left(\frac{2 c^{2}}{n_{0}^{2} \sigma}-\sigma\right)}
\end{array}\right] .
$$

It was demonstrated in Ref. [30] that with the found soliton solution in the form of Eq. (2), Eq. (1) can be converted into the nonlinear relativistic 1D Dirac equation. This analytical fundamental DS solution in BWAs is a one-parameter family where one parameter such as soliton peak amplitude or width can be arbitrary, provided that the soliton width is large enough (the beam width parameter $n_{0} \geq 3.5$, see Ref. [30] for more details). The fundamental DS solution in the form of Eq. (2) is valid in the case when $\gamma$ and $\sigma$ are positive. However, with this solution one can easily construct other Dirac soliton solutions for any sign of each parameter $\gamma$ and $\sigma$ [30].

To be specific, let us suppose that the waveguide positions of the BWA $n \in\left[-N_{1}, \ldots 0, \ldots N_{2}\right]$ with $-N_{1}$ and $N_{2}$ being two edges of the BWA. We also fix the center of the DS in the form of Eq. (2) at the waveguide position with $n=0$. The DS solution in the form of Eq. (2) is only valid if the DS is localized exclusively well inside the BWA and is much far away from both edges of the BWA. This condition is easily satisfied if $N_{1}$ and $N_{2}$ are much larger than the DS width parameter $n_{0}$. In this case, the edges of the BWA do not play any role in DS dynamics. So far, all DSs have been investigated under this condition [30,32-34]. If the DS in the form of Eq. (2) is launched into the system and its location is well inside the BWAs, then it will propagate longitudinally along BWAs without any distortion of its shape (see Fig. 2(a) in Ref. [30] for more details). It is important to emphasize that the DS in the form of Eq. (2) has two components: one weak and the other intense. These components are located adjacent to each other. To be specific, we suppose that the weak component of the DS is localized in odd waveguides, i.e., $a_{2 n+1}$ is weak, whereas the intense component is localized in even waveguides, i.e., $a_{2 n}$ is intense (see Fig. 1(a) and Fig. 2(c) in Ref. [30] for more details).

\section{EDGE EFFECTS OF TRUNCATED DIRAC SOLITONS IN BWAS}

In the rest of this work, we will explore the situation where some portion of one tail of the DS is truncated at the edge of BWAs with $N_{1}$, i.e., $N_{1}$ is comparable with $n_{0}$, whereas the other tail 
of the DS is much far away from the other edge of the BWA, i.e., $n_{0} \ll N_{2}$. We fix the DS width parameter $n_{0}=5$, and $N_{2}=200$, whereas $N_{1}$ will be varied.

Technically, the truncating process can be implemented, for instance, by placing two identical BWAs closely one after another. The full structure of the DS is generated in the $1^{\text {st }}$ BWA. The output of the beam from the $1^{\text {st }}$ BWA is coupled into the input of the $2^{\text {nd }}$ BWA. However, the $2^{\text {nd }}$ BWA can be shifted transversally so that only some part of the DS coming out from the $1^{s t}$ BWA is coupled into the $2^{\text {nd }}$ BWA, and the rest of the DS is truncated. Another way to truncate DSs can be done with just one BWA, whose one corner is removed so that at the beginning the waveguide position $n \in\left[-N_{2}, \ldots 0, \ldots N_{2}\right]$, but after some propagation the waveguide position $n \in\left[-N_{1}, \ldots 0, \ldots N_{2}\right]$. In both cases, the input center of the full DS can be shifted transversally so that one can tune the edge of the truncated DS later.

In Fig. 1(a) we show the propagation of a truncated DS in a BWA where the outermost waveguide of the BWA truncated edge has position $n=-N_{1}=-10$. This waveguide at the edge $(n=-10)$ hosts the DS intense component as clearly shown in Fig. 1(c) where we plot the input profile of the truncated DS investigated in Fig. 1(a). In Fig. 1(b) we show the propagation of a truncated DS in a BWA where the outermost waveguide of the BWA truncated edge has position $n=-N_{1}=-9$. This waveguide at the edge $(n=-9)$ hosts the DS weak component as clearly shown in Fig. 1(d) where we plot the input profile of the truncated DS investigated in Fig. 1(b). As clearly seen in Fig. 1(a) and Fig. 1(b), the dynamics of the truncated DS dramatically depends on its edge where it is truncated. If the edge of the BWA hosts the intense component of the truncated DS as in the case of Fig. 1(a) and Fig. 1(c), then, at the beginning, this truncated DS is bent towards the near edge of the BWA and touches it after reaching the propagation distance $z=L_{f}=57$. After the first touching of the truncated DS with the BWA edge, the truncated DS bounces off the BWA edge and moves towards the center region of the BWA. However, this DS movement towards the BWA center does not last forever and is stopped after some propagation, and then the truncated DS is bent towards the BWA edge again and again in a cyclic fashion. As a result, the truncated DS in Fig. 1(a) is trapped in the proximity of the BWA edge which the truncated DS is close to. On the contrary, if the edge of the BWA hosts the weak component of the truncated DS as in the case of Fig. 1(b) and Fig. 1(d), then the truncated DS is quickly pushed away from the BWA edge so that the truncated DS moves further and further from the BWA edge as clearly shown in Fig. 1(b).

In Fig. 1 the truncated edge of the DS is located at the waveguide with position $n=-N_{1}=$ -10 (Fig. 1(a) and Fig. 1(c)) and at the waveguide with position $n=-N_{1}=-9$ (Fig. 1(b) and Fig. 1(d)). So, in Fig. 1 a considerable portion of the DS is truncated. Now in Fig. 2 we will explore the situation where only a small portion of the DS tail is truncated, i.e., the truncated edge of the DS is quite far away from the DS center. We see that main features in Fig. 1 are reproduced in Fig. 2. In Fig. 2(a) we show the propagation of a truncated DS in a BWA where the DS is truncated at the waveguide with position $n=-N_{1}=-24$. Like in Fig. 1(a), this waveguide at the edge ( $n=-24)$ also hosts the DS intense component. As a result, the main feature in Fig. 1(a) is reproduced in Fig. 2(a). Indeed, the truncated DS in Fig. 2(a) is first bent towards the near edge of the BWA, then touches this edge, then bounces off this edge, and is bent towards this edge of the BWA again and again. However, the first touching distance of the truncated DS with the BWA edge in Fig. 2(a) is $z=L_{f}=851$ which is much longer than the first touching distance $\left(L_{f}=57\right)$ of the truncated DS in Fig. 1(a). This is expected, because only a small portion of the DS tail in Fig. 2(a) is truncated, whereas a considerable portion of the DS tail in Fig. 1(a) is truncated. 

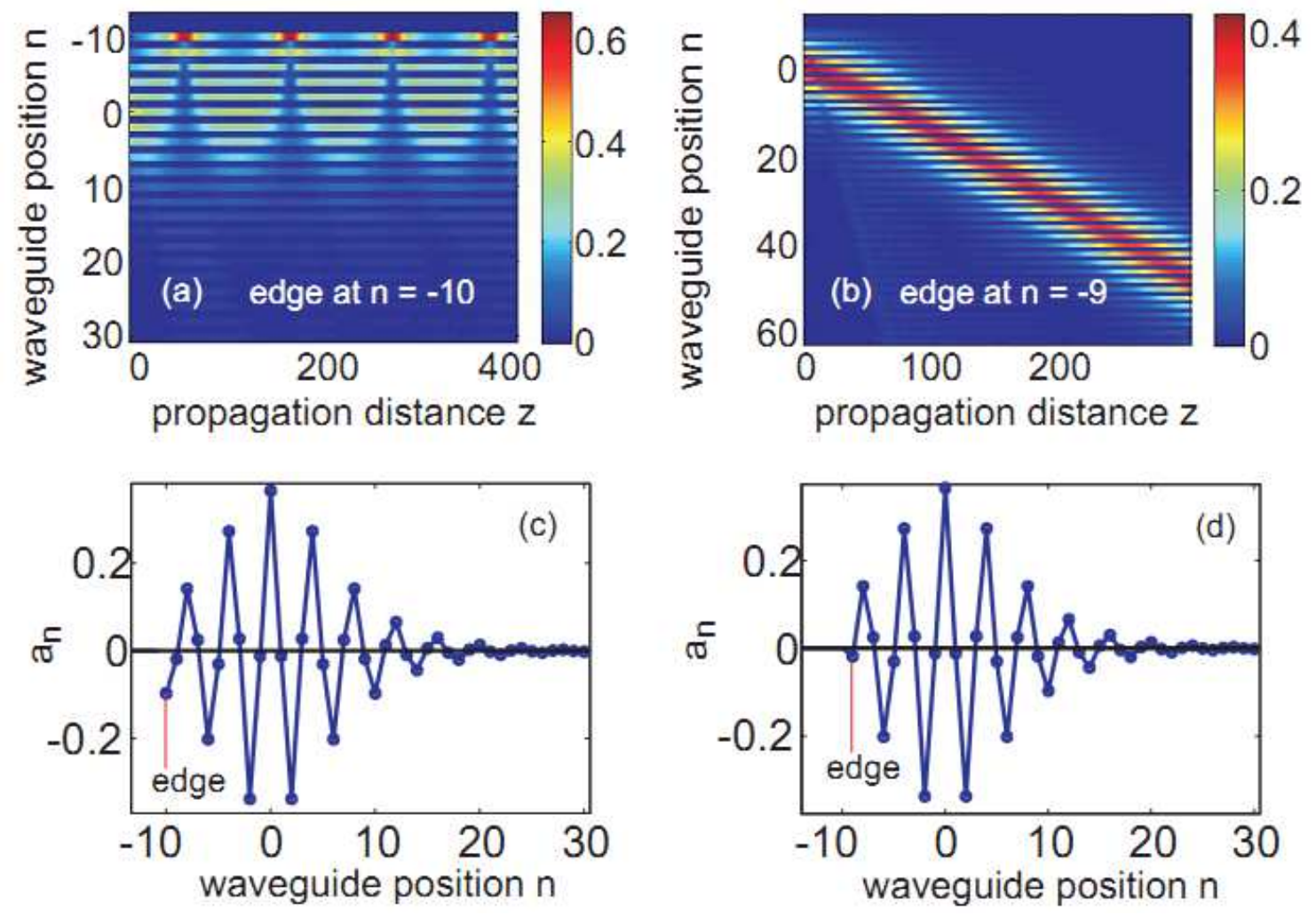

Fig. 1. (a) Propagation of a truncated DS in a BWA where the outermost waveguide at the BWA truncated edge $\left(n=-N_{1}=-10\right)$ hosts the intense component of the truncated DS. (b) Propagation of a truncated DS in a BWA where the outermost waveguide at the BWA truncated edge $\left(n=-N_{1}=-9\right)$ hosts the weak component of the truncated DS. (c) Input profile of the truncated DS shown in (a). (d) Input profile of the truncated DS shown in (b). Parameters: coupling coe?ient $c=1$, nonlinear coe?ient $\gamma=1$, propagation mismatch for the whole BWA $\sigma=-1.2$, DS width parameter $n_{0}=5$.

In Fig. 2(b) we show the propagation of a truncated DS in a BWA where the DS is truncated at the waveguide with position $n=-N_{1}=-23$. Like in Fig. 1(b), this waveguide at the edge $(n=-23)$ also hosts the DS weak component. As a result, the main feature in Fig. 1(b) is also reproduced in Fig. 2(b). Indeed, like in Fig. 1(b), the truncated DS in Fig. 2(b) is pushed away from the BWA edge so that the truncated DS moves further and further from the BWA edge as clearly shown in Fig. 2(b). The main difference between features in Fig. 1(b) and Fig. 2(b) is that the truncated DS in Fig. 2(b) is pushed away from the BWA edge in a much weaker manner so that after the propagation distance $z=1500$ the center of the DS is transversally shifted only by about 15 waveguides, whereas in Fig. 1(b) the DS center is transversally shifted by about 50 waveguides after $z=300$. This is also expected, because only a small portion of the DS tail in Fig. 2(b) is truncated, whereas a considerable portion of the DS tail in Fig. 1(b) is truncated. 

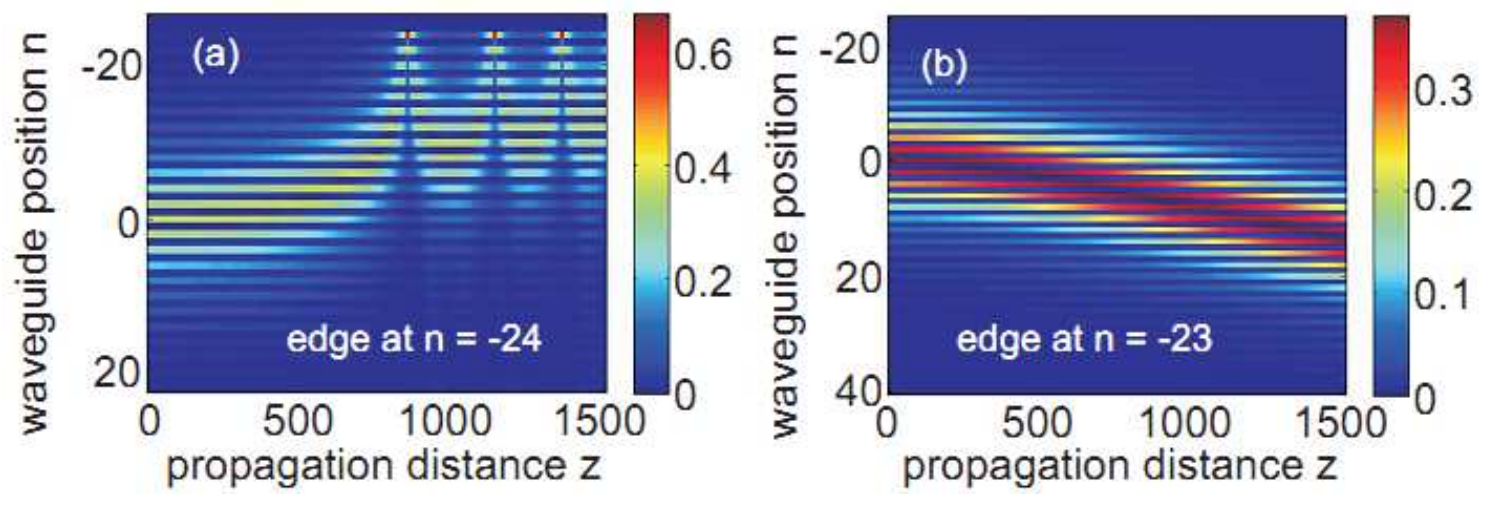

Fig. 2. (a) Propagation of a truncated DS in a BWA where the outermost waveguide at the BWA truncated edge $\left(n=-N_{1}=-24\right)$ hosts the intense component of the truncated DS. (b) Propagation of a truncated DS in a BWA where the outermost waveguide at the BWA truncated edge $\left(n=-N_{1}=-23\right.$ ) hosts the weak component of the truncated DS. All other parameters are exactly the same as in Fig. 1.

As shown above, in Fig. 2 only a small portion of the DS is truncated, whereas in Fig. 1 a considerable portion of the DS is truncated. Now in Fig. 3 we will investigate the situation where more than half a DS is truncated at the beginning. In Fig. 3(a) we show the propagation of an initially truncated DS in a BWA where the DS is truncated at the waveguide with position $n=-N_{1}$ $=2$. Note that the DS center is located at the waveguide with position $n=0$ (see Fig. 1(c)), so more than half of the DS is truncated in Fig. 3(a). Like in Fig. 1(a) and Fig. 2(a), the waveguide at the edge with position $n=2$ in Fig. 3(a) also hosts the intense component of the initially truncated DS. As a result, the initially truncated DS in Fig. 3(a) is also trapped in the proximity of the BWA edge where the truncated DS is close to. However, there is a big difference between dynamics of the initially truncated DS in Fig. 3(a) as compared to Fig. 1(a) and Fig. 2(a). Namely, in Fig. 1(a) and Fig. 2(a) one can see the distinct touching points of the truncated DS when it bounces off the BWA edge, whereas in Fig. 3(a) the initially truncated DS does not bounce off the BWA edge, but always sticks to it.

In Fig. 3(b) we show the propagation of an initially truncated DS in a BWA where the DS is truncated at the waveguide with position $n=-N_{1}=3$. Like in Fig. 3(a), more than half of the DS is truncated in Fig. 3(b) at the beginning. Like in Fig. 1(b) and Fig. 2(b), the waveguide at the edge with position $n=3$ in Fig. 3(b) also hosts the weak component of the initially truncated DS. As a result, the initially truncated DS in Fig. 3(b) is also pushed away from the BWA edge where the initially truncated DS is close to. However, there is a big difference between dynamics of the initially truncated DS in Fig. 3(b) as compared to Fig. 1(b) and Fig. 2(b). Namely, in Fig. 1(b) and Fig. 2(b) one can see that Dirac solitons still can recover their full shapes during propagation, and are well spatially localized while moving away from the near edge of the BWA. On the contrary, because much of the DS is initially truncated in Fig. 3(b), so now the initially truncated DS cannot recover its shape during propagation. Instead, it spreads out continually during propagation. As a result, the structure of the DS is completely destroyed in Fig. 3(b). In this case, it is not appropriate anymore to use the term "soliton" for this structure during propagation. 

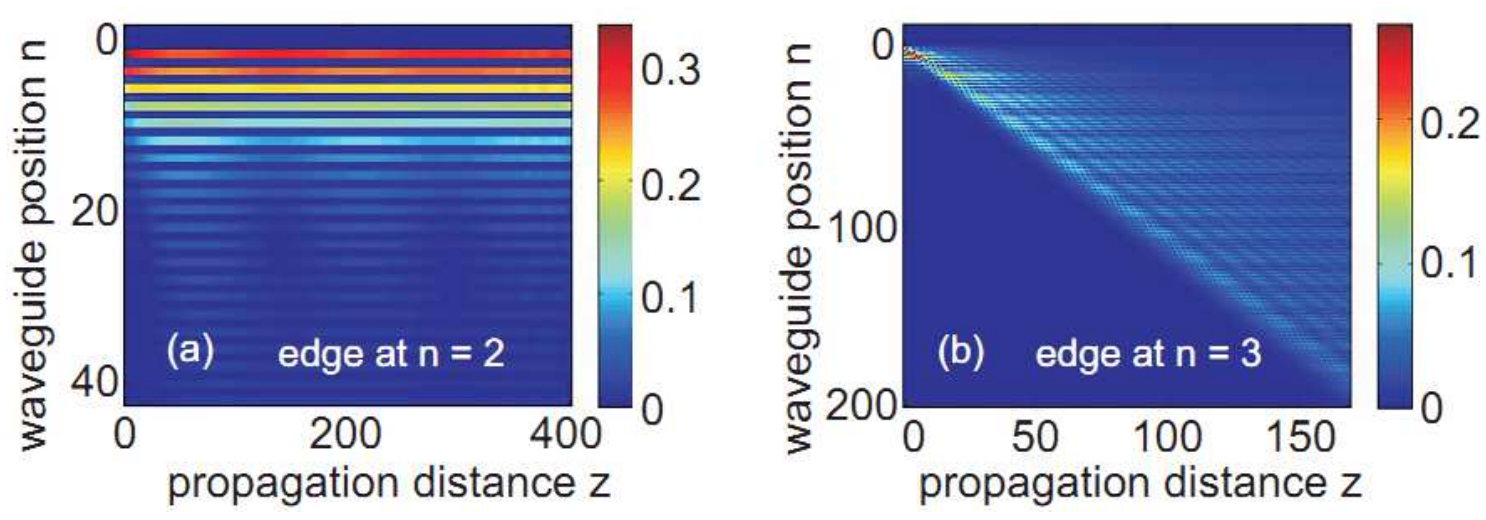

Fig. 3. (a) Propagation of an initially truncated DS in a BWA where the outermost waveguide at the BWA truncated edge $\left(n=-N_{1}=2\right)$ hosts the intense component of the truncated DS. (b) Propagation of an initially truncated DS in a BWA where the outermost waveguide at the BWA truncated edge $\left(n=-N_{1}=3\right)$ hosts the weak component of the initially truncated DS. All other parameters are exactly the same as in Fig. 1.

The physical reason why the truncated DS dynamics is so sensitive to the type of the outermost waveguide of the BWA truncated edge can be understood if one takes into account the fact that the intense component of the DS can be localized only in one type of waveguides in the BWA with smaller propagation constant (i.e., even waveguides in Eq. (1)), whereas the weak component of the DS can be localized only in the other type of waveguides in the BWA with larger propagation constant (i.e., odd waveguides in Eq. (1)) (see the last paragraph in page 181 in Ref. [30] for more details). Once the DS is truncated at one tail, the symmetry in the transverse direction of the system is broken, as a result, the truncated DS will be shifted transversally. If the outermost waveguide of the BWA truncated edge has the even position as in Fig. 1(a), Fig. 2(a) and Fig. 3(a), then this outermost waveguide can support the intense component of the DS, as a result, it is favorable for the truncated DS to move towards this outermost waveguide, and even concentrate its energy at this waveguide when it touches the edge. This scenario cannot happen if the outermost waveguide has an odd position, because in this case this type of waveguides cannot support the intense component of the DS, as a result, it is more favorable for the truncated DS to move far away from this type of the BWA edge as in Fig. 1(b), Fig. 2(b) and Fig. 3(b). It is worth mentioning that the truncated DS is only physically sensitive to the type of the outermost waveguide in the BWA truncated edge which is strictly related to its propagation constant represented by the parameter $\sigma$ in Eq. (1). In this work, to be specific, and at the same time consistent with both Eq. (1) and Eq. (2), we fix the even (odd) waveguides with the DS strong (weak) component. However, the parity of the outermost waveguide position can be mathematically easily changed by adopting another way of counting the waveguide positions in the BWA, and this will not change the physical behavior of the system.

\section{FIRST TOUCHING DISTANCE OF TRUNCATED DIRAC SOLITONS IN BWAS}

In the rest of this paper we will investigate in detail the first touching distance Lf of the truncated DS with the BWA edge which is the closest to the DS. As mentioned above, in order 
for the truncated DS to touch the BWA edge, the DS must be truncated at the waveguide position which hosts its intense component as in the case of Fig. 1(a) and Fig. 2(a). In Fig. 4 we plot the first touching distance $L_{f}$ as a function of the waveguide position at the truncated edge, i.e., $-N_{1}$. As mentioned above, when $-N_{1}=-24$ as shown in Fig. 2(a), the first touching distance $L_{f}=851$; whereas when $-N_{1}=-10$ as shown in Fig. 1(a), the first touching distance $L_{f}=57$. At the right end of the curve shown in Fig. 4 we stop at the value $-N_{1}=-2$, because if $-N_{1}=0,2,4, \ldots$ and further, then more than half of the DS is truncated which will lead to the scenario similar to the one shown in Fig. 3(a) (with $-N_{1}=2$ ) when the rest part of the truncated DS just always sticks to the BWA edge, and one cannot observe the distinguished touching points of the truncated DS. In the contrast, there is no limit for the value of $-N_{1}$ at the left end of the curve. As clearly seen in Fig. 4, the larger the absolute value of $N_{1}$, the longer the first touching distance $L_{f}$ of the truncated DS. Physically, this trend is easily explained, because when $-N_{1}$ is decreased from -2 to -26 and further, then less and less portion of the DS is truncated, as a result, the edge effect of the truncated DS will be less and less pronounced. In the limit case when $N_{1}=N_{2}$, the system is totally symmetric, the BWA is large enough, and the whole DS is quite far away from both BWA edges. In this case, of course, the DS in the form of Eq. (2) will always propagate parallel to the longitudinal axis of the BWA as shown in Fig. 2(a) in Ref. [30], and the first touching distance Lf of the DS will be infinity.

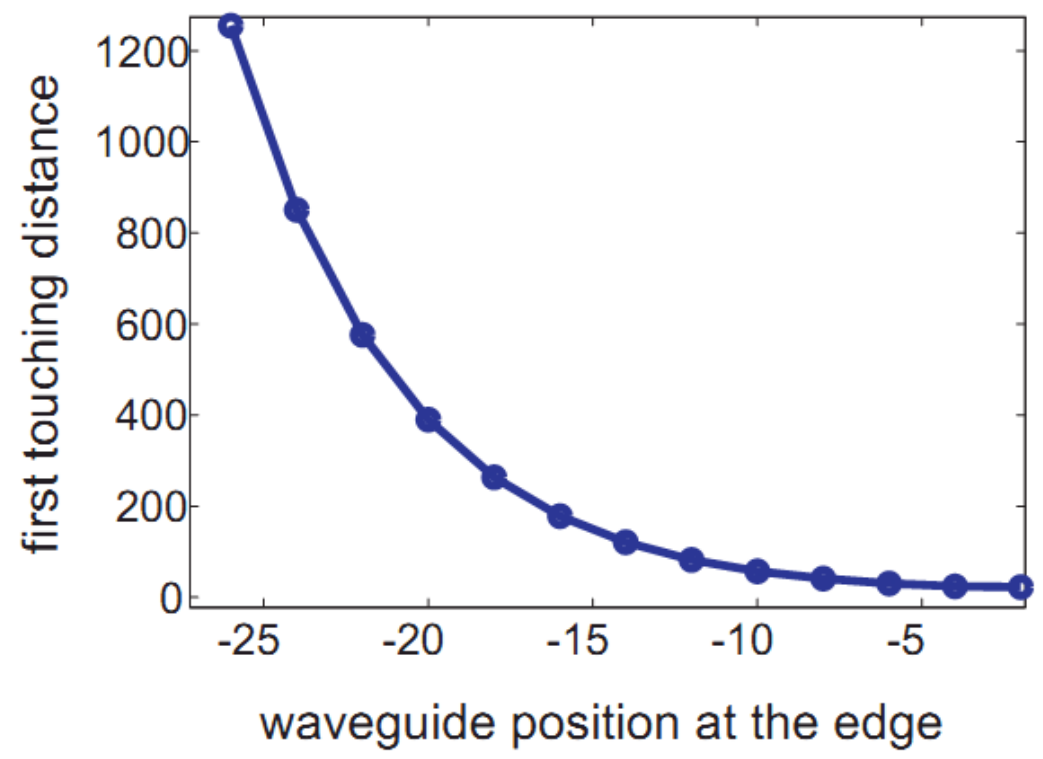

Fig. 4. The first touching distance $L_{f}$ of the truncated DS with the BWA edge as a function of the waveguide position at the truncated edge of the BWA, i.e., $-N_{1}$.

\section{CONCLUSIONS}

In conclusion, we have investigated the edge effects of the Dirac solitons in BWAs when one tail of the Dirac soliton is truncated at the input. We have numerically demonstrated that the edge effects of the truncated Dirac soliton dramatically depend on the position where the Dirac 
soliton is initially truncated. Namely, if the outermost waveguide of the BWA hosts the intense component of the truncated Dirac soliton and if less than half of the Dirac soliton is truncated at the input, then the Dirac soliton will follow the following cyclic fashion: first, it is bent towards the BWA edge, then touches it, and later bounces off the BWA edge. Meanwhile, if the outermost waveguide of the BWA hosts the intense component of the truncated Dirac soliton, but more than half of the Dirac soliton is truncated at the input, then the rest part of the truncated Dirac soliton will always stick to the BWA truncated edge. In the contrast, if the outermost waveguide of the BWA hosts the weak component of the truncated Dirac soliton, then Dirac soliton will be pushed away from the BWA edge. In the latter case, if less than half of the Dirac soliton is truncated at the input, then the Dirac soliton can recover its full shape during propagation; whereas if more than half of the Dirac soliton is truncated at the input, then the Dirac soliton cannot recover its full shape later. To the best of our knowledge, these unique features of Dirac soliton edge effects in BWAs have not been found in any other systems. These edge effects of the truncated DS in BWAs are not only interesting from the fundamental perspective, but also important from the practical point of view, because one can use them to manipulate, route, or control the DS in potential all-optical signal processing circuits.

\section{ACKNOWLEDGMENT}

This research is funded by Vietnam National Foundation for Science and Technology Development (NAFOSTED) under grant number 103.03-2016.01.

\section{REFERENCES}

[1] F. Lederer, G. I. Stegeman, D. N. Christodoulides, G. Assanto, M. Segev, and Y. Silberberg, Phys. Reports 463 (2008) 1.

[2] D. N. Christodoulides, F. Lederer, and Y. Silberberg, Nature 424 (2003) 817.

[3] T. Pertsch, P. Dannberg, W. El?ein, A. Bräuer, and F. Lederer, Phys. Rev. Lett. 83 (1999) 4752.

[4] R. Morandotti, U. Peschel, J. S. Aitchison, H. S. Eisenb erg, and Y. Silberberg, Phys. Rev. Lett. 83 (1999) 4756.

[5] M. Ghulinyan, C.J. Oton, Z. Gaburro, L. Pavesi, C. Toninelli, and D.S. Wiersma, Phys. Rev. Lett. 94 (2005) 127401.

[6] H. Trompeter, T. Pertsch, F. Lederer, D. Michaelis, U. Streppel, A. Bräuer, and U. Peschel, Phys. Rev. Lett. 96 (2006) 023901.

[7] Tr. X. Tran and F. Biancalana, Phys. Rev. Lett. 110 (2013) 113903.

[8] Tr. X. Tran and F. Biancalana, Opt. Exp. 21 (2013) 17539.

[9] Tr. X. Tran, D. C. Duong, and F. Biancalana, Phys. Rev. A 89 (2014) 013826.

[10] S. Longhi, Phys. Rev. B 81 (2010) 075102.

[11] F. Dreisow, R. Keil, A. Tünnermann, S. Nolte, S. Longhi, and A. Szameit, EPL 97 (2012) 10008.

[12] S. Longhi, Opt. Lett. 35 (2010) 235.

[13] F. Dreisow, M. Heinrich, R. Keil, A. Tünnermann, S. Nolte, S. Longhi, and A. Szameit, Phys. Rev. Lett. 105 (2010) 143902.

[14] S. Longhi, Appl. Phys. B 104 (2011) 453.

[15] J. M. Zeuner, N. K. Efremidis, R. Keil, F. Dreisow, D. N. Christodoulides, A. Tünnermann, S. Nolte, and A. Szameit, Phys. Rev. Lett. 109 (2012) 023602.

[16] Tr. X. Tran and F. Biancalana, Phys. Rev. A 96 (2017) 013831.

[17] R. Jackiw and C. Rebbi, Phys. Rev. D 13 (1976) 3398.

[18] R.B. Laughlin, "Nobel Lecture: Fractional quantization", Rev. Mod. Phys. 71 (1999) 863.

[19] M.Z. Hasan and C.L. Kane, Rev. Mod. Phys. 82 (2010) 3045.

[20] X.L. Qi and S.C. Zhang, Rev. Mod. Phys. 83 (2011) 1057. 
[21] M.C. Rechtsman, J. M. Zeuner, Y. Plotnik, Y. Lumer, D. Podolsky, F. Dreisow, S. Nolte, M. Segev, and A. Szameit, Nature (London), 496 (2013) 196.

[22] L. Lu, J.D. Joannopoulos, and M. Soljaic, Nat. Photonics 8 (2014) 821.

[23] A. A. Sukhorukov and Y. S. Kivshar, Opt. Lett. 27 (2002) 2112.

[24] A. A. Sukhorukov and Y. S. Kivshar, Opt. Lett. 28 (2003) 2345.

[25] M. Conforti, C. De Angelis, and T.R. Akylas, Phys. Rev. A 83 (2011) 043822.

[26] M. Johansson, K. Kirr, A. S. Kovalev, and L. Kroon, Physica Scripta 83 (2011) 065005.

[27] A. Gorbach and M. Johansson, Eur. Phys. J. D 29 (2004) 77.

[28] M. Johansson and A. Gorbach, Phys. Rev. E 70 (2004) 057604.

[29] R. Morandotti, D. Mandelik, Y. Silberberg, J. S. Aitchison, M. Sorel, D. N. Christodoulides, A.A. Sukhorukov, and Y. S. Kivshar, Opt. Lett. 29 (2004) 2890.

[30] Tr. X. Tran, S. Longhi, and F. Biancalana, Ann. Phys. 340 (2014) 179.

[31] Y. Nogami, F.M. Toyama, and Z. Zhao, J. Phys. A: Math. Gen. 28 (1995) 1413.

[32] Tr. X. Tran, X. N. Nguyen, and D. C. Duong, J. Opt. Soc. Am. B 31 (2014) 1132.

[33] Tr. X. Tran, X. N. Nguyen, and F. Biancalana, Phys. Rev. A 91 (2015) 023814.

[34] Tr. X. Tran and D. C. Duong, Ann. Phys. 361 (2015) 501.

[35] W. Heisenberg, Rev. Mod. Phys. 29 (1957) 269.

[36] D. C. Ionescu, R. Reinhardt, B. Muller, and W. Greiner, Phys. Rev. A 38 (1988) 616.

[37] A. Zecca, Internat. J. Theoret. Phys. 41 (2002) 421.

[38] M. J. Esteban and E. Séré, Discrete Contin. Dyn. Syst. 8 (2002) 381.

[39] I. Bialynicki-Birula and J. Mycielski, Ann. Phys. 100 (1976) 62.

[40] N. Kemmer, Helv. Phys. Acta 10 (1937) 47.

[41] E. Fermi and C.N. Yang, Phys. Rev. 76 (1949) 1739.

[42] G.P. Agrawal, Nonlinear Fiber Optics, 5th ed. (Academic, 2013).

[43] Y. S. Kivshar and G.P. Agrawal, Optical Solitons: from Fiber to Photonic Crystals, 5th ed. (Academic, 2003).

[44] R. Morandotti, U. Peschel, J. S. Aitchison, H. S. Eisenberg, and Y. Silberberg, Phys. Rev. Lett. 83 (1999) 2726.

[45] P. G. Kevrekidis and M.I. Weinstein, Math. Comput. Simul. 62 (2003) 65.

[46] G. P. Agrawal, Applications of Nonlinear Fiber Optics, 2nd ed. (Academic, 2008).

[47] Tr. X. Tran, D. C. Duong, and F. Biancalana, Phys. Rev. A 90 (2014) 023857. 